\title{
Interplay of magnetic and structural transitions in Fe-based pnictide superconductors
}

\author{
A. Cano, ${ }^{1}$ M. Civelli, ${ }^{2}$ I. Eremin,${ }^{3}$ and I. Paul ${ }^{4}$ \\ 1 European Synchrotron Radiation Facility, 6 rue Jules Horowitz, BP 220, 38043 Grenoble, France \\ ${ }^{2}$ Institut Laue-Langevin, 6 rue Jules Horowitz, BP 156, 38042 Grenoble, France \\ ${ }^{3}$ Institut für Theoretische Physik III, Ruhr-Universität Bochum, 44801 Bochum, Germany \\ 4 Institut Néel, CNRS/UJF, 25 avenue des Martyrs, BP 166, 38042 Grenoble, France
}

(Dated: November 1, 2018)

\begin{abstract}
The interplay between the structural and magnetic phase transitions occurring in the Fe-based pnictide superconductors is studied within a Ginzburg-Landau approach. We show that the magnetoelastic coupling between the corresponding order parameters is behind the salient features observed in the phase diagram of these systems. This naturally explains the coincidence of transition temperatures observed in some cases as well as the character (first or second-order) of the transitions. We also show that magnetoelastic coupling is the key ingredient determining the collinearity of the magnetic ordering, and we propose an experimental criterion to distinguish between a pure elastic from a spin-nematic-driven structural transition.

PACS numbers: 74.70.Xa, 74.90.+n, 75.80.+q
\end{abstract}

Introduction.- The discovery of an unconventional high-temperature superconductivity in the F-doped arsenic-oxide $\mathrm{LaFeAsO}^{1}$ has given rise to a great interest on iron pnictide compounds. To date several families of Fe-based superconductors have been discovered, and the superconducting transition temperature has been raised to above $50 \mathrm{~K} .^{2}$ All of these systems display an intriguing competition between structural, magnetic and superconducting transitions. ${ }^{2,3}$ The parent compounds of the so-called 1111 and 111 families undergo a structural transition (ST) followed by a magnetic transition (MT) at a lower temperature, ${ }^{4}$ whereas in the 122 and 11 cases these two transitions take place simultaneously. ${ }^{5-7}$ In any case these orderings are quickly suppressed by doping or by applying pressure, which eventually gives rise to superconductivity. The role, if any, of magnetic and elastic degrees of freedom in inducing this superconductivity is currently an open question. There is already a growing body of theoretical works advocating for spin fluctuation mediated superconductivity, ${ }^{8}$ but the isotope effect observed in both magnetism and superconductivity suggests that the elastic medium also plays a role. ${ }^{9}$ It is therefore compelling to understand the connection between the ST and the MT in these systems.

The ST, for example, has been studied in Refs. 10,11 where it has been identified with a spin-nematic ordering, but ignoring the possible softening of the lattice itself. This idea has been further elaborated in Ref. 12, where the observed softening of the lattice is interpreted as due to the fluctuations of the emerging nematic degrees of freedom. Recent studies ${ }^{13}$ have also associated the ST with a possible ordering of the $d_{x y}$ and $d_{y z}$ Fe-orbitals in the Fe-As planes, with the consequent distortions having an impact on both the magnetic order ${ }^{13,14}$ and the electronic structure. ${ }^{15}$ On the other hand, the study of the MT in Ref. 16 focuses on the role of the magnetoelastic (ME) couplings in producing a weak first-order MT by means of the Larkin-Pikin mechanism. ${ }^{17}$ This latter is relevant only in the fluctuation dominated Ginzburg regime and is subjected to the condition of a MT with diverging specific heat in the absence of ME coupling. Furthermore, the collinearity of the magnetic moments is examined in Ref. 18 by using a purely electronic model.

In this paper we study the ST and the MT using a Ginzburg-Landau approach, in which the interplay between elastic and magnetic degrees of freedom is considered explicitly. This provides a general unified framework that goes beyond previous phenomenological approaches and rationalizes different experimental findings. We address, in particular, the simultaneity of the ST and MT, their character (first versus second order), the collinearity of the magnetic structure, and the spin-nematic scenario for the ST. Our study identifies a particular ME coupling [see Eq. (2) below] as the common key factor behind the salient features of the ST and MT in the Fe-based superconductors.

Our main results are the following. (i) We derive the general phase diagram of the ST and MT, which exhibits four qualitatively different regimes, as shown in Fig. 1. This explains why the simultaneous ST and MT in the 122 compounds are sometimes observed as second-order transitions $^{6}$ (Ia in Fig. 1) and sometimes as first-order (Ib in Fig. 1). We moreover predict a first-order MT when this occurs separate but in the immediate vicinity of the ST (IIa in Fig. 1). This richness of the phase diagram is due to the ME coupling. (ii) The collinearity of the magnetic moments is linked to the existence of a particular ME term allowed by symmetry. (iii) An experimental criterion is derived to distinguish between a pure elastic from a spin-nematic-driven ST. (iv) The fluctuations associated with ST are shown to become critical only along certain lines of high symmetry in the Brillouin zone. Along these lines the sound velocity vanishes in a pure elastic ST or the spin-nematic excitations are massless in a spin-nematic-driven ST. In both scenarios the ST has a mean-field behavior with no divergences. ${ }^{11}$

Ginzburg-Landau free energy.- The basic ingredients to build up a Ginzburg-Landau theory for the ST and the 


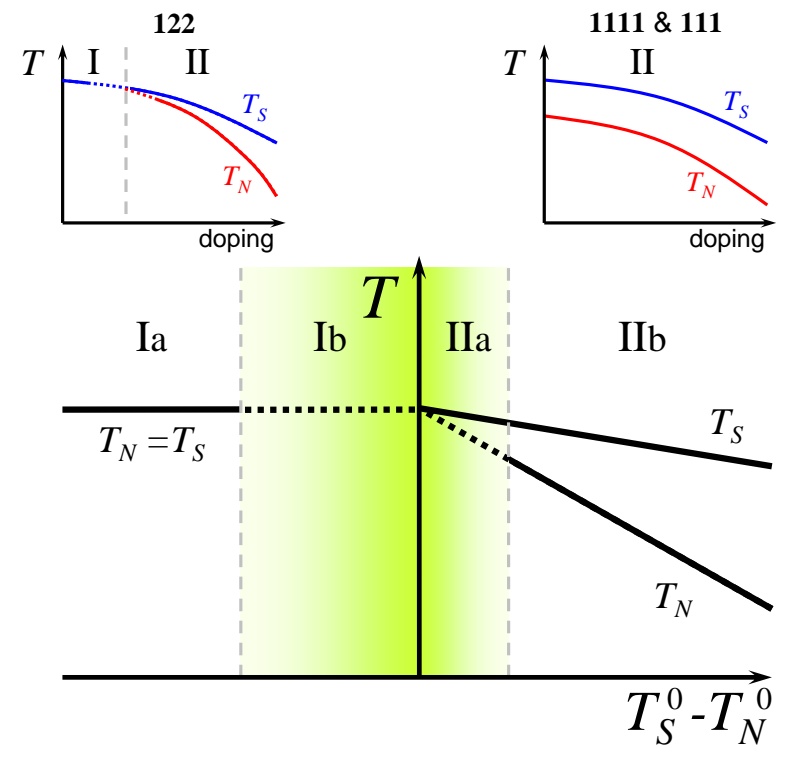

FIG. 1: Schematic $T$ vs. doping diagrams of the possible structural and magnetic phase transitions in Fe-based superconductors (top panels) and general phase diagram $T$ vs. $T_{S}^{0}-T_{N}^{0}$ determined by our Ginzburg-Landau theory Eq. (1) (bottom panel). Empirically $T_{S}^{0}-T_{N}^{0}$ increases with doping. In the regions I the structural and magnetic are simultaneous, while in regions II they take place at different transition temperatures, $T_{S}$ and $T_{N}$ respectively. Continuous (dotted) lines indicate second (first) order phase transitions. The strength of the first order discontinuity is indicated by the intensity of the background color.

MT in the Fe-based superconductors are the following. The initial order parameter for the ST can be taken as the shear strain $u_{x y},{ }^{19}$ where subscripts refer to the principal axes of the tetragonal lattice (with two and four Fe atoms per unit cell for the 1111 and the 122 systems respectively, see Fig. 2a). Thus $u_{x y} \neq 0$ implies the monoclinic distortion of the tetragonal unit cell observed experimentally, as illustrated in Fig. 2b. For the case of a spin-nematic driven ST an additional order parameter $\sigma$ is required, which will be explained in more detail below. On the other hand the description of the MT requires two Néel vectors, ${ }^{20} \mathbf{L}_{1}$ and $\mathbf{L}_{2}$, which can be associated with the magnetizations $\mathbf{M}_{1}$ and $\mathbf{M}_{2}$ of the two inter-penetrating Fe sublattices (Fig. 2b). The Ginzburg-Landau free energy then can be conveniently written as

$$
F_{G L}=F_{M}+F_{E}+F_{M E}
$$

Here the magnetic part is $F_{M}=\frac{1}{2} A\left(L_{1}^{2}+L_{2}^{2}\right)+\frac{1}{4} B\left(L_{1}^{4}+\right.$ $\left.L_{2}^{4}\right)$. In principle fourth order terms of the form $L_{1}^{2} L_{2}^{2}$ and $\left(\mathbf{L}_{1} \cdot \mathbf{L}_{2}\right)^{2}$ are also allowed by symmetry, but we omit them because the former does not affect the results qualitatively while the latter is generated by the ME coupling (see below). For the elastic part we consider explicitly the critical strain $u_{x y}$ only and we write $F_{E}=\frac{1}{2} c_{66} u_{x y}^{2}+\frac{1}{4} \beta u_{x y}^{4}$. The coefficients $A$ and $c_{66}$ are as- (a)

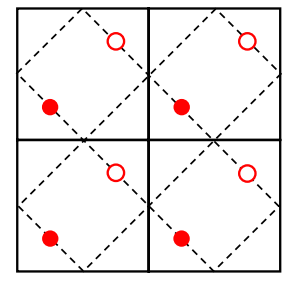

(b)

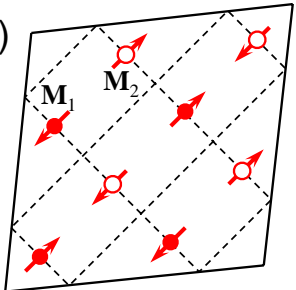

FIG. 2: Tetragonal Fe-lattice (drawn as two interpenetrating sublattices) at temperatures $T>T_{S}, T_{N}$ (a). A monoclinic distortion of the tetragonal unit cell and a collinear Néel order in the two Fe sublattices take place at $T<T_{S}, T_{N}$ (b).

sumed to vary with the temperature as $A=A^{\prime}\left(T-T_{N}^{0}\right)$ and $c_{66}=c_{66}^{\prime}\left(T-T_{S}^{0}\right) . \quad T_{N}^{0}$ and $T_{S}^{0}$ are the nominal MT and ST temperatures respectively (not to be confused with the actual transition temperatures $T_{N}$ and $\left.T_{S}\right)$, taken as the control parameters of our theory. It is our empirical obsevation that the results of the LandauGinzburg theory are consistent with the current experiments if we assume that $T_{S}^{0}-T_{N}^{0}$ increses with doping. A possible expalantion of this trend at very low doping is the following. It is likely that the effect of doping is more on the magnetic sector (say, via the loss of nesting between electron and hole bands) than the elastic medium which it affects indirectly. Thus, it may not be unreasonable to expect that at low doping $T_{S}^{0}$ stays relatively unchanged while $T_{N}^{0}$ decreases (since the system hardens magnetically). The remaining coefficients $B, C$ and $\beta$ are taken as positive constants. As regards the ME term, the key contribution is ${ }^{11,12,16}$

$$
F_{M E}=g_{1} u_{x y}\left(\mathbf{L}_{1} \cdot \mathbf{L}_{2}\right),
$$

where $g_{1}>0$ in order to be consistent with the experimental observation that the ferromagnetic Fe-Fe bonds are shorter than the antiferromagnetic ones in the collinear-Néel state. To our purposes, the standard magneto-striction $u_{l l}\left(L_{1}^{2}+L_{2}^{2}\right)$ can be neglected because it does not change qualitatively the results. It is worth mentioning that, in the context of the cuprate superconductors, a similar free energy $F_{G L}$ was used to study the structural transition of doped $\mathrm{La}_{2} \mathrm{SrCuO}_{4} \cdot{ }^{21}$

Phase diagram.- We are in a position to discuss the general phase diagram for the ST and MT in the Febased superconductors (Fig. 1). We anticipate that, experimentally, undoped and lightly doped 122 systems fall in regimes Ia and Ib with simultaneous transitions of second and first order respectively. On the other hand, the 1111 and 111 compounds appear to fall in the regime IIb, where the transitions are separate $\left(T_{S}>T_{N}\right)$ and second order. In the regime IIa the ST is second order while the separate MT is first order. This regime has not yet been reported experimentally, perhaps because it is narrow in the tuning of physical parameters (such as pressure and doping) and therefore difficult to detect.

Within our framework, the fact that these transitions sometimes are observed separate and sometimes simulta- 
neous is explained as follows. Note that, once the magnetic order sets in and the collinear-Néel state is formed, there is an effective shear stress that produces a monoclinic distortion via the ME term (Eq. 2). Thus, the $\mathrm{MT}$ in these systems implies a ST, while the converse is evidently not true. This gives rise to the regimes I and II in the phase diagram shown in Fig. 1, which are defined by the conditions $T_{S}^{0}<T_{N}^{0}$ and $T_{S}^{0}>T_{N}^{0}$ respectively. Thus, as the temperature is lowered in regime II there is first the $\mathrm{ST}$ at $T=T_{S}^{0}$, where $c_{66}=0$, followed by a separate MT at $T_{N}=T_{N}^{0}+\left[g_{1} u_{0}\left(T_{N}\right)\right] / A^{\prime}$, where $u_{0}(T)= \pm\left[\left(T_{S}^{0}-T\right) c_{66}^{\prime} / \beta\right]^{1 / 2}$ is the value of the strain order parameter $u_{x y}$ in the monoclinic phase at $T<T_{S}$. In the regime I, however, the system encounters the MT first at $T=T_{N}^{0}$, where $A=0$, but simultaneously the ST occurs due to the effective stress in the ME term. In this regime, unlike in II, there is no instability of the lattice because the elastic modulus $c_{66}$ stays finite (and jumps across the transition).

These two regimes are divided in their turn into subregimes $\mathrm{Ia}, \mathrm{Ib}$, IIa and $\mathrm{IIb}$ according to the character, first or second order, of the MT. This is again due to the ME coupling, and has nothing to do with symmetry reasons (such as the existence of cubic invariants). The point is that the effective coefficient of fourth-order term in the magnetic sector can become negative due to the ME coupling (in which case, one has to include higher order terms to bound the free energy from below). This is readily seen in the regime $\mathrm{I}\left(T_{S}^{0}<T_{N}^{0}\right)$, where the elastic sector is stable and one can ignore the $u_{x y}^{4}$ term in $F_{E}$. Indeed minimizing $F_{G L}$ one gets $u_{x y}=-g_{1}\left(\mathbf{L}_{1} \cdot \mathbf{L}_{2}\right) / c_{66}$, which implies that the elastic sector generates an effective magnetic term of the form $-g_{1}^{2}\left(\mathbf{L}_{1} \cdot \mathbf{L}_{2}\right)^{2} /\left(2 c_{66}\right)$. The MT into the collinearNéel state turns first order when this term is sufficiently strong, with the tri-critical point given by $c_{66}=g_{1}^{2} / B$ and $A=0$. The logic is quite similar in the regime II $\left(T_{S}^{0}>T_{N}^{0}\right)$, where one first has to expand $u_{x y}=u_{0}+\delta u_{0}$ in the monoclinic phase to then obtain (after further minimization) $\delta u_{0}=-g_{1}\left(\mathbf{L}_{1} \cdot \mathbf{L}_{2}\right) /\left(2\left|c_{66}\right|\right)$. This implies an effective magnetic term $-g_{1}^{2}\left(\mathbf{L}_{1} \cdot \mathbf{L}_{2}\right)^{2} /\left(4\left|c_{66}\right|\right)$ which drives the MT first order, with the tri-critical point given by $A=g_{1} u_{0}\left(T_{N}\right)$ and $c_{66}=-g_{1}^{2} /(2 B)$. These results have to be contrasted to Ref. 16 , where strong fluctuations are required and weaker first-order transitions, with narrower hysteresis, are expected. At the mean field level, it is the vicinity of the structural instability (where $c_{66}=0$ ) to determine the area (region IIa in the phase diagram of Fig. 1) where the MT becomes first order due to the ME coupling. In fact the discontinuity is the stronger the closer is to the point $T_{S}^{0}=T_{N}^{0}$ (as indicated by the color intensity in Fig. 1).

Collinearity of the magnetic order.- At this stage, the role of the $\mathrm{ME}$ coupling in favoring the collinearNéel state observed experimentally ${ }^{22}$ is quite straightforward. In regime II the collinearity is due to the term $g_{1} u_{0}\left(\mathbf{L}_{1} \cdot \mathbf{L}_{2}\right)$, which lifts the degeneracy of the transition temperatures for $\mathbf{L}_{ \pm}=\frac{1}{\sqrt{2}}\left(\mathbf{L}_{1} \pm \mathbf{L}_{2}\right)$. This implies that only one between $\mathbf{L}_{ \pm}$is non-zero in the ordered state (which one in particular depends on the sign of $u_{0}$, i.e., on the ferroelastic domain). In regime I, however, the reason is different. In this case the set up of the collinear order is associated with the negative coefficient $-g_{1}^{2} /\left(2 c_{66}\right)$ in front of the term $\left(\mathbf{L}_{1} \cdot \mathbf{L}_{2}\right)^{2}$, generated by the ME coupling. Presumably, the vicinity to the structural instability makes this ME term strong enough to overcome any other contribution (due to, e.g., magnetic interactions) and thus forces the collinearity.

Spin nematic versus pure elastic instability. - In the region II of the phase diagram, where the two transitions are separate, there are two different physical scenarios to conceptualize the ST: a pure ferroelastic transition or a transition induced by a spin-nematic ordering. In the following we discuss some physical implications that may allow to determine experimentally which one of the two scenario is realized in the Fe-based superconductors.

(a) In the first scenario we have a proper ferroelastic instability in $F_{E}$ caused by the vanishing of the elastic modulus $c_{66}$ (as described above). An immediate consequence is that the temperature range over which the monoclinic distortion has square-root behavior $u_{0}(T) \propto\left(T_{S}-T\right)^{1 / 2}$ is the same as the temperature range over which the elastic modulus is expected to have a linear dependence $c_{66} \propto\left|T-T_{S}\right|$.

(b) The second scenario is more subtle, as it arises from the possibility that, above $T_{N}$, the system enters a spin nematic state in which the two Néel vectors are zero in average but fluctuate in phase, i.e., $\sigma \equiv\left\langle\mathbf{L}_{1} \cdot \mathbf{L}_{2}\right\rangle \neq 0 .{ }^{23}$ In this case the system is expected to undergo a pseudoproper ferroelastic transition driven by the nematic ordering. This scenario can be described by the free energy

$$
\widetilde{F}_{G L}=\frac{a}{2} \sigma^{2}+\frac{b}{4} \sigma^{4}+\frac{c_{66}}{2} u_{x y}^{2}+\frac{\beta}{4} u_{x y}^{4}+g_{2} u_{x y} \sigma,
$$

where $a=a^{\prime}\left(T-T_{n}^{0}\right)$ with $T_{n}^{0}$ being the nominal nematic transition temperature, while all other coefficients are $T$-independent (including $c_{66}$ for this discussion). The structural-nematic transition, below which $u_{x y} \propto \sigma \neq 0$, occurs at $T_{S}=T_{n}^{0}+g_{2}^{2} /\left(c_{66} a^{\prime}\right)$ where the effective elastic modulus $\widetilde{c}_{66}=c_{66}-g_{2}^{2} / a$ vanishes. In this case, the range of temperatures over which $\widetilde{c}_{66} \propto\left|T-T_{S}\right|$ is restricted to the condition $\left|T-T_{S}\right|<g_{2}^{2} /\left(c_{66} a^{\prime}\right)$. This range is in principle different from the $T$-range over which the monoclinic distortion has square-root dependence, as we have discussed in point (a) above.

This simple observation, which is schematically represent in Fig. 3, can be used as a quantitative criterion to distinguish between the pure ferroelastic and spin-nematic-induced scenarios. These considerations are based on a mean field analysis of the ST, which we justify in the following.

Mean-field behavior for the structural transition.- Previous studies on ferroelastics ${ }^{24}$ have shown that a secondorder ST, like in the proper ferroelastic scenario (a), displays a mean-field behavior. This follows from the fact that at the transition point, where the elastic mod- 


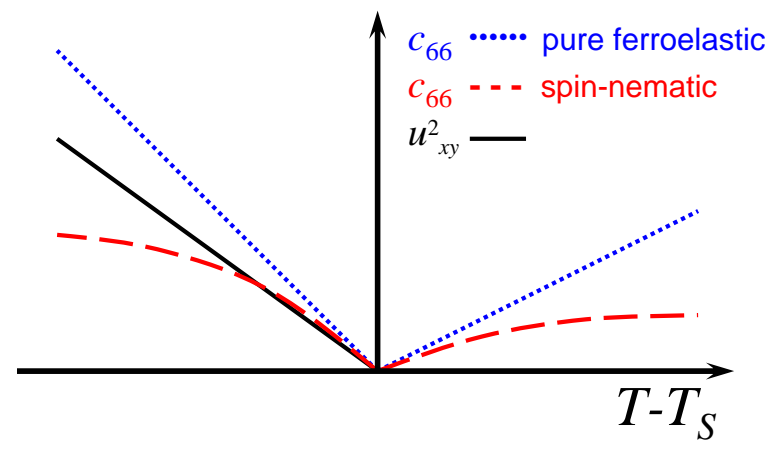

FIG. 3: Expected dependence on temperature of the squared spontaneous strain $u_{x y}^{2}$ and the elastic modulus $c_{66}$ in scenarios (a) proper ferroelastic instability and (b) driven by spinnematic ordering. Large deviations from a linear behavior in $c_{66}$ as compared to $u_{x y}^{2}$ indicate spin-nematic ordering.

ulus $c_{66}=0$, the phonon velocity remains finite everywhere in the Brillouin zone (q-space), except along the two lines of high symmetry $q_{x}=0$ and $q_{y}=0$ on the $q_{z}=0$ plane. Consequently, except for these "soft" lines, the long-wavelength critical excitations associated with the strain field $u_{x y}$ are gapped and the transition in three dimensions is mean-field like. ${ }^{24}$ The contribution of these excitations to $F_{E}$ can be written as $\delta F_{E}=\frac{1}{2} \sum_{\mathbf{q} \neq 0} c_{66}(\mathbf{q})\left|u_{x y}(\mathbf{q})\right|^{2}+\cdots$, where the ellipsis denotes non-critical modes and interaction terms,

$$
c_{66}(\mathbf{q}) \approx c_{66}+c_{1} \cos ^{2} \theta+c_{2} \sin ^{2} 2 \varphi \sin ^{4} \theta+\kappa q^{2}
$$

near the soft lines, and $\theta$ and $\varphi$ represent the polar and the azimuthal angles respectively in q-space. ${ }^{24}$ The $\kappa q^{2}$ contribution is due to harmonic terms in the free energy with higher order derivatives (the usual stiffness associated with collective excitations) and $c_{1}$ and $c_{2}$ are constants that depend on the elastic moduli of the system. ${ }^{25}$ In the scenario (b) the situation is slightly more subtle. Indeed, if we ignore the coupling $g_{2} u_{x y} \sigma$, it appears that the nematic transition, which belongs to the Ising class, is in three dimensions below its upper critical dimension and therefore it is not mean-field type. However, the ME coupling gives rise to anisotropic correc- tions to the mass of the $\sigma$-field. In fact the resulting mass term can be written as $F_{\sigma}=\frac{1}{2} \sum_{\mathbf{q} \neq 0} a(\mathbf{q})|\sigma(\mathbf{q})|^{2}$, where $a(\mathbf{q})=a-g_{2}^{2} / c_{66}(\mathbf{q})$. As a consequence, in this scenario too, the long-wavelength critical excitations are gapped except along two soft lines. ${ }^{26}$ So the ST is completely mean field in any case.

We finally discuss some limitations of our study. We have restricted ourselves to magnetic states describable in terms of the Néel vectors $\mathbf{L}_{1}$ and $\mathbf{L}_{2}$. This includes the collinear $(\pi, 0)$ [or $(0, \pi)$ ] "stripe" order observed experimentally. In the case of a different magnetic ground state the corresponding Ginzburg-Landau theory may have a different form, with structural and magnetic orderings not necessarily coupled. This seems to be the case in $\mathrm{BaMn}_{2} \mathrm{As}_{2}$, where a G-type antiferromagnetic order is found and no structural distortion is observed. ${ }^{27}$ The same applies to 11 Fe-chalcogenides, for which, in addition, the question of whether the magnetic order is intrinsic or arises from non-stoichiometry, i.e., due to interstitial Fe-ions inducing weak charge localization, is still open. ${ }^{28}$ Critical fluctuations are also not considered in our theory. While we expect that they will not change the nature of the ST, they certainly will enhance the effects of the Larkin-Pikin mechanism ${ }^{17}$ on the MT. We do expect therefore an enlargement of the first order MT region (Ib and IIa in Fig. 1). All these considerations deserve deeper investigation in future studies.

In summary, we have shown that the magneto-elastic coupling Eq. (2) plays a key role in producing the specific features of the structural and magnetic transitions in the Fe-based superconductors. This coupling is responsible for the simultaneity of the transitions observed in the 122 systems and explains the different characters (first-order and second-order) of the transitions reported so far. It also naturally explains the collinearity of the magnetic order. We have also addressed the question of the possibility of a spin-nematic driven structural transition, indicating an experimental way to discriminate this type of scenario from a proper ferroelastic transition.

We acknowledge G. Garbarino, E. Kats, M. NúñezRegueiro, J. Schmalian and T. Ziman for stimulating discussions.
1 Y. Kamihara et. al., J. Am. Chem. Soc. 130, 3296 (2008).

2 See, e.g., M.D. Lumsden and A.D. Christianson, arXiv:1004.1969

3 See, e.g., M.R. Norman, Physics 1, 21 (2008).

4 See, e.g., M.A. McGuire et. al., Phys. Rev. B 78, 094517 (2008); G. Garbarino et al., Phys. Rev. B 78, 100507(R) (2008); S. Li, et. al., Phys. Rev. B 80, 020504(R) (2009);

5 T. Yamazaki et. al., arXiv:1003.0913; Z. Li, et al., arXiv:1004.4328.

6 S.D. Wilson et. al., Phys. Rev. B 79, 184519 (09).

7 S. Li et al., Phys. Rev. B 79, 054503 (2009); W. Bao et al., Phys. Rev. Lett. 102, 247001 (2009).
8 See, e.g., I.I. Mazin and J. Schmalian, Physica C 469, 614 (2009).

9 R.H. Liu et. al., Nature 459, 64 (2009).

10 C. Fang et. al., Phys. Rev. B 77, 224509 (2008); C. Xu, M. Müller, and S. Sachdev, Phys. Rev. B 78, 020501(R) (2008).

11 Y. Qi and C. Xu, Phys. Rev. B 80, 094402 (2009).

12 R.M. Fernandes et al., arXiv:0911.3084.

13 W. Lv, J. Wu, and P. Phillips, Phys. Rev. B 80, 224506 (2009); A. M. Turner, Fa Wang, and A. Vishwanath, Phys. Rev. B 80, 224504, C.-C. Lee, W.-G. Yin, and W. Ku, Phys. Rev. Lett. 103, 267001 (2009). 
14 F. Krüger, S. Kumar, J. Zaanen, and J. van den Brink, Phys. Rev. B 79, 054504 (2009); M. Daghofer et al., arXiv:1004.4803.

15 W. Lv, F. Krüger, and P. Phillips, arXiv:1002.3165, M.J. Calderon, B. Valenzuela and E. Bascones, New J. Phys. 11, 013051 (2009).

16 V. Barzykin and L.P. Gor'kov, Phys. Rev. B 79, 134510 (2009).

17 A.I. Larkin and S.A. Pikin, Zh. Eksp. Teor. Fiz. 56, 1664 (1969) [Sov. Phys. JETP 29, 891 (1969)].

18 I. Eremin, A.V. Chubukov, Phys. Rev. B 81, 024511 (2010).

19 The strain tensor is defined as $u_{i j}=\frac{1}{2}\left(\partial_{i} u_{j}+\partial_{j} u_{i}\right)$, where $\mathbf{u}$ is the displacement vector in a continuum medium description.

20 The two sublattice magnetizations associated with the $(\pi, 0)$ [or $(0, \pi)]$ orderings observed experimentally can be defined as $\mathbf{M}_{1}=\mathbf{L}_{1} \cos \left(\mathbf{Q} \cdot \mathbf{R}_{1}\right) \cos (\pi z)$ and $\mathbf{M}_{2}=$ $\mathbf{L}_{2} \cos \left(\mathbf{Q} \cdot \mathbf{R}_{2}\right) \cos (\pi z)$, where $\mathbf{Q}=(\pi, \pi)$ and $\mathbf{R}_{1(2)}$ are points on the Fe sublattice 1(2). The observed moments are $\sim 0.3-0.7 \mu_{B}$ in the 1111 and 111 and $\sim 1.5 \mu_{B}$ in the 122 compounds.

21 J.L. Sarrao et. al., Phys. Rev. B 50, 13125 (1994).

${ }^{22}$ See, e.g., C. de la Cruz et. al., Nature 453, 899 (2008).

23 P. Chandra, P. Coleman and A.I. Larkin, Phys. Rev. Lett. 64, 88 (1990).

24 R.A. Cowley, Phys. Rev. B 13, 4877 (1976); R. Folk, H. Iro and F. Schwabl, Z. Phys. B 25, 69 (1976).

25 If, for example, $F_{E}=\frac{\lambda}{2} u_{l l}^{2}+\mu u_{i j}^{2}+\frac{1}{2}\left(c_{66}-4 \mu\right) u_{x y}^{2}$ where $\lambda$ and $\mu$ are the Lame coefficients, one has $c_{1}=4 \mu$ and $c_{2}=16 \mu(\lambda+\mu) /(\lambda+2 \mu)$. The most general form of $F_{E}$ with tetragonal symmetry involves six elastic constants, but, for convenience's sake, we use a simplified model with only three elastic constants.

26 A.P. Levanyuk and A.A. Sobyanin, Pis'ma Zh. Eksp. Teor. Fiz. 11, 540 (1970) [JETP Lett. 11, 371 (1970)].

27 Y. Singh et al., arXiv:0907.4094.

28 see e.g. T.J. Liu et al., arXiv:1003.5647. 